\section{Mutations in the desmosomal protein plakophilin-2 are common in arrhythmogenic right ventricular cardiomyopathy}

\author{
Brenda Gerull ${ }^{1,2,7}$, Arnd Heuser ${ }^{1,2,7}$, Thomas Wichter ${ }^{3}$, \\ Matthias Paul ${ }^{3}$, Craig T Basson ${ }^{4}$, Deborah A McDermott ${ }^{4}$, \\ Bruce B Lerman ${ }^{4}$, Steve M Markowitz ${ }^{4}$, Patrick T Ellinor ${ }^{5}$, \\ Calum A MacRae ${ }^{5}$, Stefan Peters ${ }^{6}$, Katja S Grossmann ${ }^{1}$, \\ Beate Michely ${ }^{1,2}$, Sabine Sasse-Klaassen ${ }^{1}$, \\ Walter Birchmeier ${ }^{1}$, Rainer Dietz ${ }^{2}$, Günter Breithardt ${ }^{3}$, \\ Eric Schulze-Bahr ${ }^{3}$ \& Ludwig Thierfelder ${ }^{1,2}$
}

Arrhythmogenic right ventricular cardiomyopathy (ARVC) is associated with fibrofatty replacement of cardiac myocytes, ventricular tachyarrhythmias and sudden cardiac death. In 32 of 120 unrelated individuals with ARVC, we identified heterozygous mutations in PKP2, which encodes plakophilin-2, an essential armadillo-repeat protein of the cardiac desmosome. In two kindreds with ARVC, disease was incompletely penetrant in most carriers of PKP2 mutations.

Individuals with ARVC may present with palpitations or ventricular tachyarrhythmias that originate from the right ventricle and may lead to syncope or sudden cardiac death, a relatively common and the most feared clinical manifestation of the disease ${ }^{1-3}$. Localized or diffuse atrophy and progressive replacement of right ventricular myocytes with fatty or fibrofatty tissue is the pathological hallmark of ARVC. Although right ventricular involvement predominates, the left ventricle also can be affected. Heart failure of the right and, less commonly, the left ventricle may occur later in the disease process. The exact prevalence and incidence of ARVC is unknown. Familial disease accounts for an unknown proportion of ARVC, with estimates ranging from $30 \%$ to $80 \%$. Eight genomic loci and rare mutations in plakoglobin $(J U P)$, desmoplakin $(D S P)$ and the cardiac ryanodine receptor (RYR2) have been reported in nonsyndromic and syndromic cases ${ }^{2}$.

Desmosomes are complex multiprotein structures of the cell membrane and provide structural and functional integrity to adjacent cells (e.g., epithelial cells and cardiomyocytes ${ }^{4}$ ). Desmosomal proteins also have a role in cell signaling, ${ }^{5,6}$. At least three groups of molecules contribute to the formation of desmosomes: desmosomal cadherins, armadillo-repeat proteins and plakins ${ }^{7}$. The plakophilins, which are armadillo-related proteins, contain ten 42-amino acid armadillorepeat motifs and are located in the outer dense plaque of desmosomes linking desmosomal cadherins with desmoplakin and the intermediate filament system ${ }^{8}$. Like other armadillo-repeat proteins, plakophilins are also found in the nucleus, where they may have a role in transcriptional regulation ${ }^{9}$. Plakophilin-2 exists in two alternatively spliced isoforms ( $2 \mathrm{a}$ and $2 \mathrm{~b}$ ), interacts with multiple other cell adhesion proteins and is the primary cardiac plakophilin ${ }^{8,10}$.

On the basis of findings of a lethal defect in cardiac morphogenesis at embryonic day 10.75 in mice homozygous with respect to a deletion mutation of $P k p 2$ (ref. 11), we hypothesized that mutations in human PKP2 may account for ARVC. A total of 120 unrelated probands of western European descent (101 males and 19 females) were admitted to tertiary referral centers and diagnosed with ARVC in accordance with clinical criteria proposed by a Task Force ${ }^{12}$. We directly sequenced all 14 PKP2 exons, including flanking intronic splice sequences (Supplementary Methods online), and identified 25 different heterozygous mutations in 32 probands $(27$ males and 5 females). Of the 25 PKP2 mutations, 12 were insertion-deletion mutations, 6 were nonsense mutations, 4 were missense mutations and 3 were splice site mutations (Table 1 and Supplementary Figs. 1 and 2 online). Although most mutations were detected in the C-terminal half of the molecule, mutations were located throughout the gene. We observed one PKP2 splice acceptor site mutation $(2146-1 \mathrm{G} \rightarrow \mathrm{C})$ and two nonsense mutations $(235 \mathrm{C} \rightarrow \mathrm{T}$ and $2203 \mathrm{C} \rightarrow \mathrm{T}$ ) in several unrelated individuals with ARVC (Table 1). Genetic analysis of microsatellite DNA markers at or near the PKP2 locus on chromosome $12 \mathrm{p} 11$ did not identify shared haplotypes between the ARVC cases with identical mutations (data not shown). Because parental genotypes were not available, however, we could not carry out formal segregation analyses. Therefore, ancient founder mutations cannot be excluded with certainty. Six instances of a nonsense mutation in PKP2 $(235 \mathrm{C} \rightarrow \mathrm{T})$ indicated that nucleotide residue 235 might be a mutational hot spot. None of the 25 PKP2 mutations were observed in 500 control chromosomes.

Although familial disease was not systematically evaluated in all probands, members of two kindreds (kindreds A100 and EPF; Fig. 1) were available for a detailed clinical and molecular analysis. A 2-bp deletion in PKP2 exon 10 (2076_2077delAA) is predicted to cause the addition of 48 amino acid residues before a premature stop signal is introduced (C693fsX741); we observed this mutation in two clinically affected members of kindred A100 (individuals 18 and 161; Fig. 1 and Supplementary Table 1 online). We carried out western-blot analysis

\footnotetext{
${ }^{1}$ Max-Delbrüeck Center for Molecular Medicine, D-13092 Berlin-Buch, Germany. ${ }^{2}$ Department of Clinical and Molecular Cardiology, Franz-Volhard Clinic, HELIOS Clinics GmbH, Charité, Humboldt University Berlin, D-13125 Berlin, Germany. ${ }^{3}$ Department of Cardiology and Angiology, University Hospital of Münster, and the Institute for Arteriosclerosis Research at the University of Münster, D-48149 Münster, Germany. ${ }^{4}$ Greenberg Cardiology Division, Dept. of Medicine, Weill Medical College of Cornell University, New York, New York 10021, USA. ${ }^{5}$ Cardiac Arrhythmia Service and Cardiovascular Research Center, Massachusetts General Hospital, Charlestown, Massachusetts 02129, USA. ${ }^{6}$ Department of Cardiology, Klinikum Quedlinburg, D-06484 Quedlinburg, Germany. ${ }^{7}$ These authors contributed equally to this work. Correspondence should be addressed to L.T. (Ithier@mdc-berlin.de).
} 
Table 1 Clinical status and PKP2 mutations of 32 index individuals with ARVC

\begin{tabular}{|c|c|c|c|c|c|}
\hline Individual & Sex & Clinical status & Exon & Nucleotide change & Amino acid change \\
\hline 1 & $M$ & VT, RV, LV, H & 1 & 145_148delCAGA & S50fsX110 \\
\hline EPF17 & $\mathrm{F}$ & $\mathrm{RV}, \mathrm{H}$ & 1 & 216insG & Q74fsX85 \\
\hline 3 & $\mathrm{M}$ & VT, RV, H & 2 & $235 \mathrm{C} \rightarrow \mathrm{T}$ & R79X \\
\hline 4 & M & VT, RV & 2 & $235 \mathrm{C} \rightarrow \mathrm{T}$ & R79X \\
\hline 5 & M & $\mathrm{S}, \mathrm{CA}, \mathrm{VT}, \mathrm{RV}$ & 2 & $235 \mathrm{C} \rightarrow \mathrm{T}$ & R79X \\
\hline 6 & $\mathrm{M}$ & $\mathrm{CA}, \mathrm{VT}, \mathrm{RV}, \mathrm{LV}$ & 2 & $235 \mathrm{C} \rightarrow \mathrm{T}$ & R79X \\
\hline 7 & $\mathrm{M}$ & VT, RV & 2 & $235 \mathrm{C} \rightarrow \mathrm{T}$ & R79X \\
\hline 8 & M & VT, RV & 2 & $235 \mathrm{C} \rightarrow \mathrm{T}$ & R79X \\
\hline 9 & $\mathrm{~F}$ & VT, RV & 3 & $419 \mathrm{C} \rightarrow \mathrm{T}$ & S140F \\
\hline 10 & M & VT, RV & 3 & 534_535insCT & C179fsX190 \\
\hline 11 & $\mathrm{M}$ & VT, RV, LV & 5 & 1369_1372deICAAA & Q457X \\
\hline 12 & $M$ & VT, RV & 7 & 1631_1632insTT & L544fsX563 \\
\hline 13 & $\mathrm{M}$ & S, VT, RV, LV & 7 & 1642delG & V548fsX562 \\
\hline 14 & $\mathrm{M}$ & $\mathrm{S}, \mathrm{VT}, \mathrm{RV}, \mathrm{LV}$ & 9 & $1844 \mathrm{C} \rightarrow \mathrm{T}$ & S615F \\
\hline 15 & $\mathrm{M}$ & $\mathrm{S}, \mathrm{VT}, \mathrm{RV}$ & 9 & $1912 \mathrm{C} \rightarrow \mathrm{T}$ & Q638X \\
\hline 16 & M & $\mathrm{S}, \mathrm{VT}, \mathrm{RV}, \mathrm{LV}, \mathrm{H}$ & 9 & 1948delA & V650fsX655 \\
\hline 17 & $M$ & VT, RV, LV, H, HTX & 9 & $1951 C \rightarrow T$ & R651X \\
\hline 18 & M & $\mathrm{S}, \mathrm{VT}, \mathrm{RV}$ & 9 & $1960 A \rightarrow C$ & K654Q \\
\hline A100/161 & M & VT, RV, H & 10 & 2076_2077delAA & C693fsX741 \\
\hline 20 & $\mathrm{M}$ & S, RV & 10 & 2088insA & K696fsX742 \\
\hline 21 & M & $\mathrm{S}, \mathrm{VT}, \mathrm{RV}$ & 10 & $2095 C \rightarrow T$ & Q699X \\
\hline 22 & M & $\mathrm{CA}, \mathrm{VT}, \mathrm{RV}, \mathrm{H}$ & 11 & $2146-1 G \rightarrow C$ & Mutant splice product* \\
\hline 23 & M & $\mathrm{S}, \mathrm{VT}, \mathrm{RV}, \mathrm{LV}$ & 11 & $2146-1 G \rightarrow C$ & Mutant splice product* \\
\hline 24 & $\mathrm{M}$ & VT, RV & 11 & $2176 \mathrm{C} \rightarrow \mathrm{T}$ & Q726X \\
\hline 25 & $\mathrm{~F}$ & VT, RV, LV, H & 11 & $2203 C \rightarrow T$ & R735X \\
\hline 26 & $\mathrm{M}$ & $\mathrm{S}, \mathrm{VT}, \mathrm{RV}$ & 11 & $2203 C \rightarrow T$ & $\mathrm{R} 735 \mathrm{X}$ \\
\hline 27 & M & VT, RV & 12 & $2386 \mathrm{~T} \rightarrow \mathrm{C}$ & C796R \\
\hline 28 & $\mathrm{~F}$ & VT, RV & 12 & 2393_2401deICATTGAACA & N798fsX879 \\
\hline 29 & M & $\mathrm{S}, \mathrm{SCD}, \mathrm{VT}, \mathrm{RV}, \mathrm{LV}, \mathrm{H}$ & 12 & 2424insA & E809fsX826 \\
\hline 30 & $\mathrm{~F}$ & VT, RV & 12 & $2489+1 G \rightarrow A$ & Mutant splice products \\
\hline 31 & M & $\mathrm{S}, \mathrm{VT}, \mathrm{RV}, \mathrm{LV}$ & 13 & $2490-1 G \rightarrow C$ & Mutant splice products \\
\hline 32 & M & VT, RV, LV & 13 & 2509delA & V837fsX930 \\
\hline
\end{tabular}

N Male; F, female; RV, right ventricular involvement; LV, left ventricular involvement; H, positive family history;

(2) S, syncope; CA, cardiac arrest; HTX, heart transplantation. *See Supplementary Figure 2 online. Numbering of the nucleotides starts at the ATG codon of PKP2.

of a right ventricular biopsy specimen obtained from individual A100/161 but did not detect the truncated plakophilin-2 protein (expected size of truncated PKP2, $82.1 \mathrm{kDa}$; normal PKP2, 97.4 $\mathrm{kDa})$. The specimen did contain less wild-type plakophilin-2 than control myocardium (Supplementary Fig. 3 online), implicating haploinsufficiency as the operant mechanism in this PKP2 mutation.

Clinical evaluation of first-degree relatives of kindred A100 identified two individuals (individuals 18 and 161; Fig. 1 and Supplementary Table 1 online) who are affected by ARVC in accordance with the Task Force criteria ${ }^{12}$. Although four other members of kindred A100 also carried the 2-bp deletion mutation in PKP2, they have normal or mild disease phenotypes, even at more advanced ages (e.g., individual 1). Two members of another family with ARVC (EPF) died suddenly at ages 16 years and 15 years (individuals 11 and 12; Fig. 1). An autopsy study showed that both individuals had ARVC. Of the five surviving mutation carriers in kindred EPF, only one showed clinical manifestations diagnostic of ARVC (individual EPF/17; Supplementary Table 1 online). The reason for this incomplete penetrance in kindreds A100 and EPF is unknown, but it may be related to gender, genetic or epigenetic modifiers or other unknown factors (e.g., viral infection as trigger for disease). Although transmission of disease in kindreds A100 and EPF is compatible with autosomal dominance, linkage analysis would have been difficult due to incomplete penetrance of disease in both kindreds $(<0.5)$. ARVC in kindred EPF has been previously reported to be linked to chromosome 2q32.1-q32.3 (ref. 13). The identification of mutation 216insG in PKP2 in this kindred, coupled with the absence in any individual family of statistical significance for the original linkage to chromosome 2, implies that assignment of ARVC to chromosome 2 on the basis of aggregation of lod scores from three small families should possibly be considered erroneous.

Plakophilins, together with the other desmosomal proteins, assemble to form cell adhesion complexes that carry out diverse structural and functional tasks. These include mechanically safeguarding cellular and organ architecture by transmitting force between cells and participating in several signal transduction pathways. Support for the idea that plakophilins have an essential role in desmosome formation and function comes from this and two other reports: (i) the identification of recessive mutations in $P K P 1$ that cause ectodermal dysplasia/skin fragility syndrome, a rare skin disorder characterized by small, poorly formed desmosomes and perturbed desmosome-keratin intermediate filament interactions ${ }^{14}$; and (ii) the ablation of mouse $P k p 2$, the main plakophilin in cardiac muscle, which results in defects of heart morphogenesis and junctional architecture with embryonic lethality at midgestation ${ }^{11}$. Therefore, plakophilin-1 and plakophilin-2 have essential roles in desmosome formation and in skin and heart development, respectively.

How PKP2 mutations perturb cardiac desmosome assembly and function in ARVC is unknown. We speculate that lack of plakophilin-2 or incorporation of mutant plakophilin-2 into cardiac desmosomes impairs cell-cell contacts and, as a consequence, disrupts adjacent cardiomyocytes, particularly in response to mechanical stress or stretch (thus providing a potential explanation for the high prevalence of the disorder in athletes, the frequent occurrence of ventricular tachyarrhythmias and sudden death during exercise and the predominant affection of the right ventricle). Intercellular disruption would occur first in areas of high stress and stretch: the right ventricular outflow tract, apex and inferobasal (subtricuspid) area, which are pathological predilection areas in ARVC (forming the 'triangle of dysplasia' ${ }^{15}$ ). The potential cellular mechanism for the initiation of ventricular tachyarrhythmias in ARVC is the intrinsic variation in conduction properties as a result of these patchy areas of fibrofatty myocyte degeneration.

Because mutations causing ARVC have been identified in PKP2 (plakophilin-2), JUP (plakoglobin) and DSP (desmoplakin), ARVC may be considered as a disease of the desmosome.

All genetic analyses were done after informed consent was obtained from the participating individuals. The local ethical committees from Humboldt University Berlin, University of Münster, Weill Medical College of Cornell University and Harvard University approved the experimental plan. 


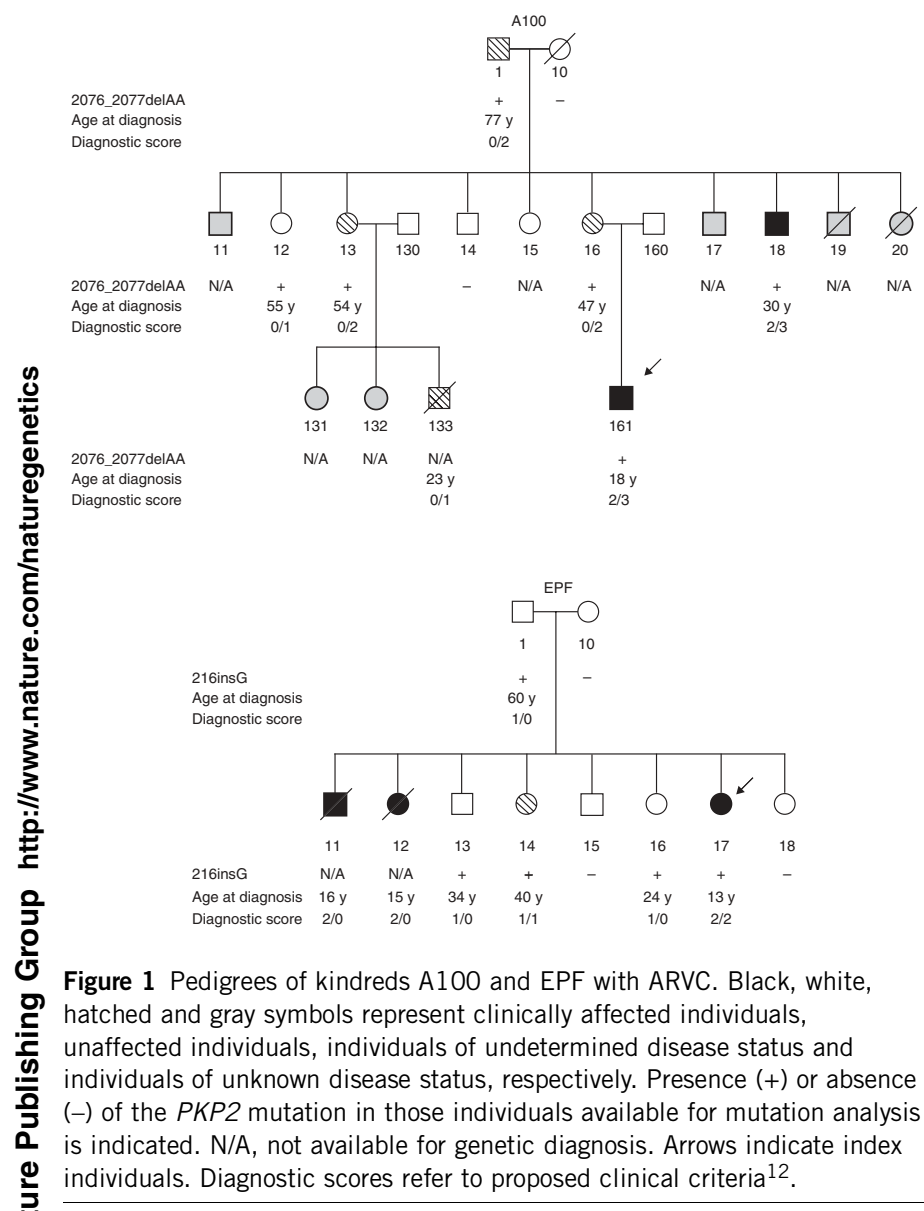

GenBank accession number. $P K P 2, \mathrm{X} 97675$.

Note: Supplementary information is available on the Nature Genetics website.

\section{ACKNOWLEDGMENTS}

We thank I. Trippmacher, S. Milan, E. Schulze-Bahr, P. Gerdes, S. Pereira and S. Helms for technical help; J. Waigand for carrying out the cardiac biopsies; and J. Schulz-Menger, B. Pilz, B. Keweloh, B. Struk, A. Schirdewan and H. Peters for logistic help. This work was supported by grants from the Ernst und Berta Grimmke Stiftung, Düsseldorf, Germany (L.T.); the Deutsche Forschungsgemeinschaft, Bonn, Germany (T.W.); the Fondation Leducq, Paris, France (E.S.-B.); the European Commission, Brussels, Belgium (T.W.); and the National Heart, Lung and Blood Institute, National Institutes of Health, Bethesda, Maryland, USA (C.T.B.).

\section{COMPETING INTERESTS STATEMENT}

The authors declare that they have no competing financial interests.

Received 28 June; accepted 28 September 2004

Published online at http://www.nature.com/naturegenetics/

1. Thiene, G., Nava, A., Corrado, D., Rossi, L. \& Pennelli, N. N. Engl. J. Med. 318, 129 133 (1988).

2. Paul, M., Schulze-Bahr, E., Breithardt, G. \& Wichter, T.A. Z. Kardiol. 92, 128-136 (2003).

3. Fontaine, G. et al. Annu. Rev. Med. 50, 17-35 (1999).

4. Green, K.J. \& Gaudry, C.A. Nat. Rev. Mol. Cell Biol. 1, 208-216 (2000).

5. Jamora, C. \& Fuchs, E. Nat. Cell Biol. 4, E101-E108 (2002).

6. Ko, K.S., Arora, P.D. \& McCulloch, C.A. J. Biol. Chem. 276, 35967-35977 (2001).

7. Garrod, D.R., Merritt, A.J. \& Nie, Z. Curr. Opin. Cell Biol. 14, 537-545 (2002).

8. Mertens, C., Kuhn, C. \& Franke, W.W. J. Cell Biol. 135, 1009-1025 (1996).

9. Mertens, C. et al. Proc. Natl. Acad. Sci. USA 98, 7795-7800 (2001).

10. Chen, X., Bonne, S., Hatzfeld, M., van Roy, F. \& Green, K.J. J. Biol. Chem. 277, 10512-10522 (2002).

11. Grossmann, K. et al. J. Cell Biol. (in the press).

12. McKenna, W.J. et al. Br. Heart J. 71, 215-218 (1994).

13. Rampazzo, A. et al. Genomics 45, 259-263 (1997).

14. McGrath, J.A. et al. Nat. Genet. 17, 240-244 (1997).

15. Marcus, F.I. et al. Circulation 65, 384-398 (1982). 